\section{RMD Open}

Rheumatic \&

Musculoskeletal Diseases

\title{
ADA2 deficiency: case report of a new phenotype and novel mutation in two sisters
}

\author{
F Uettwiller, ${ }^{1,2}$ G Sarrabay, ${ }^{3}$ M P Rodero, ${ }^{4}$ G I Rice, ${ }^{5}$ E Lagrue,${ }^{6,7}$ Y Marot, ${ }^{8}$ \\ K Deiva, ${ }^{9}$ I Touitou, ${ }^{3}$ Y J Crow, ${ }^{4,5}$ P Quartier $^{1}$
}

To cite: Uettwiller $F$,

Sarrabay G, Rodero MP, et al. ADA2 deficiency: case report of a new phenotype and novel mutation in two sisters. RMD Open 2016;2:e000236. doi:10.1136/rmdopen-2015000236

- Prepublication history and additional material is available. To view please visit the journal (http://dx.doi.org/ 10.1136/rmdopen-2015000236).

Received 19 December 2015 Revised 14 April 2016 Accepted 16 April 2016

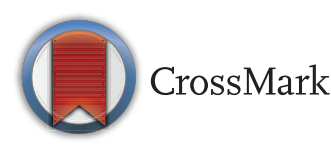

For numbered affiliations see end of article.

Correspondence to Dr F Uettwiller; florence.uettwiller@aphp.fr

\section{ABSTRACT}

The objective of this paper is to: describe the phenotype compound heterozygote for mutations in CECR1 in two children. We describe the clinical and immunological phenotype, including the assessment of ADA2 activity, cytokine expression, interferonstimulated and neutrophil-stimulated gene signatures, and the results of CECR1 sequencing. The first patient presented with intermittent fever, cutaneous vasculitis, myalgia and muscle inflammation on MRI leading to a provisional diagnosis of periarteritis nodosa.

Subsequently, two cerebral lacunar lesions were identified following a brain stroke. Clinical features improved on anti-tumour necrosis factor therapy. The first patient's sister demonstrated early-onset, longlasting anaemia with mild biological inflammation; at the ages of 3 and 5 years, she had presented 2 acute, transient neurological events with lacunar lesions on MRI. CECR1 sequencing identified both sisters to be compound heterozygous for a p.Tyr453Cys mutation and a previously undescribed deletion of exon 7. ADA2 activity was reduced by $50 \%$. Neutrophil-stimulated genes were not overexpressed, but interferonstimulated genes were. The expression of a panel of other cytokine transcripts was not significantly altered. In conclusion, searching for CECR1 mutation or assessing ADA2 activity should be considered in patients with an atypical presentation of inflammatory disease.

\section{INTRODUCTION}

Loss-of-function mutations in CECR1 have recently been described in patients with vascular and inflammatory features ranging from early-onset recurrent strokes to systemic vasculitis mimicking polyarteritis nodosa. ${ }^{1} 2$ While the pathological basis of ADA1 deficiency is relatively well understood, the pathogenesis of ADA2 deficiency remains unclear. ${ }^{3}{ }^{4}$ A previous report suggested a possible link to an overexpression of neutrophilderived gene transcripts. ${ }^{5}$ We describe two new cases of ADA2 deficiency in siblings with

\section{Key messages}

What is already known about this subject?

ADA2 deficiency is associated with various phenotypes, from early-onset inflammation mimicking periarteritis nodosa to central nervous system involvement; cutaneous lesions are considered a key clinical feature.

\section{What does this study add?}

- This study extends the phenotypic spectrum of ADA2 deficiency to atypical myositis and unexplained neurological events in the absence of cutaneous involvement.

- Gene expression may show an interferon 1 sig nature but not necessarily the previously described neutrophilic-associated gene overexpression.

- Our study emphasises the need to search for CECR1 exonic deletions in suspected cases.

How might this impact on clinical practice?

- In patients with persistent, early-onset inflammation, even in the absence of the most typical features, ADA2 deficiency should be considered and anti-tumour necrosis factor therapy discussed.

an unusual presentation, and an assessment of ADA2 activity, cytokine secretion and gene expression profiling.

\section{MATERIALS AND METHODS}

The clinical description of the two siblings was based on the medical data. Appropriate informed consent was obtained from the parents.

ADA2 activity in plasma was assessed twice, with a 3-month interval, using a commercial kit (Diazyme Laboratories). Genomic DNA was extracted from peripheral blood leukocytes using standard procedures. Exons 2-10 of CECR1 (NM_001282225.1) and flanking intronic sequences were amplified by PCR 
(Applied Biosystems). Real-time quantitative PCR was performed for each coding exon, using a LightCycler 480 (Roche). Variants were analysed using standard in silico tools (SIFT, Polyphen2, MutationTaster). Neutrophils, interferon (IFN) and a panel of other cytokine transcripts were analysed as described previously ${ }^{5}$ (see online supplementary method).

\section{RESULTS}

Patient 1: A 9-year-old girl had presented at the age of 3.5 years with fever, cutaneous vasculitis and myalgia. Biological analyses demonstrated inflammation on both C reactive protein (CRP) $(100 \mathrm{mg} / \mathrm{L})$ and erythrocyte sedimentation rate (ESR) $(40 \mathrm{~mm})$, elevated antinuclear antibodies (ANA 1/800), a transient lupus anticoagulant, low IgM and IgA level, mild CD19 B cells, CD8 T cells and natural killer (NK) lymphopenia. Nailfold capillaroscopy showed no vessels due to a severe vasculitis. Muscle testing was abnormal with a predominant proximal muscle weakness. The creatine kinase level was normal. Muscle MRI and positron emission tomography (PET) scan revealed inflammatory lesions of the limbs (figure 1A). Muscle biopsy identified focal necrotising angiitis compatible with periarteritis nodosa.

At the age of 5 years, she had presented with two episodes of severe vertigo, vomiting and encephalopathy. Cerebral MRI was normal, but a cerebrospinal fluid (CSF) pleocytosis was identified (16 lymphocytes $/ \mathrm{mm}^{3}$ ). Clinical symptoms and vertigo improved on $2 \mathrm{mg} / \mathrm{kg}$ of prednisone. Following steroid treatment withdrawal, a cutaneous vasculitis appeared, necessitating the introduction of cyclosporine. The patient then developed an ileitis and cyclosporine was switched to azathioprine.

At the age of 7 years, a period of 2 months of persistent headache had been followed by an encephalopathy and, a few weeks later, despite the increase of steroids, by left internuclear ophthalmoplaegia. Cerebral MRI with diffusion was again reported as normal. Treatment was changed to cyclophosphamide, but the patient then developed ataxia with right body hemiparesis, left facial paresis and aphasia, 2 months later. MRI showed gadolinium-enhancing lesions with subtle abnormality diffusion sequences suggestive of a vascular stroke (figure 1B), which was later confirmed by their evolution into lacunar lesions a few months later (figure 1C). During follow-up, the patient developed moderate hypertension. Despite immunosuppressive therapy, her pyramidal syndrome worsened and systemic inflammation persisted. After switching to anti-tumour necrosis factor (anti-TNF) therapy (etanercept), the biological abnormalities resolved, with no further evidence of disease activity 1 year later. The recent MRI showed the occurrence of another asymptomatic lacunar lesion of the internal capsule (figure 1C).

Patient 2: The younger sister had been followed for uncharacterised microcytic anaemia since the age of 1 year, with a mild inflammatory syndrome of unknown origin. IgM and IgA levels were low, with mild CD19 B lymphopenia. ANA was positive $(1 / 200)$. The patient had neither cutaneous, visceral nor muscle signs, nor fever. At the age of 3 years, she presented with a transient paralysis of the right extrinsic third cranial nerve, with persistent systemic inflammation. Cerebral MRI with diffusion sequences and CSF were normal. At the age of 5 years she presented again, with transient gait disturbance, unexplained fever and mild biological inflammation. A provisional diagnosis of viral vestibulitis was made. Cerebral MRI showed a $2 \mathrm{~mm}$ lacunar stroke of the internal capsule. CSF was again normal. Treatment with anti-TNF was started, associated with a rapid normalisation of the inflammation and no other symptoms over the following year.

CECR1 sequencing revealed a heterozygous c.1358A $>$ G; p.Tyr453Cys mutation in both siblings (figure 2A). This variant is predicted to be pathogenic by in silico analysis, and has been described previously. ${ }^{1}$ Furthermore, real-time quantitative PCR revealed a heterozygous deletion of exon 7 (c.973-?_1081+?del; p.Val325Thrfs*7), predicted to lead to a frameshift and truncated protein in both children (figure 2B). Parental analysis (father's genotype: c.[973-? _1081+?del];[=]; mother's genotype: c.[1358A>G];[=]) confirmed that the sisters were compound heterozygous for these two lesions.

ADA2 activity was measured on two occasions and was consistently reduced to $50 \%$ of wild-type levels in both children (figure 2C).

IFN and neutrophil signatures were evaluated in unstimulated patient cells from whole blood samples. The expression of a panel of IFN-stimulated genes was significantly elevated in both patients (figure 3A), though for the older sister the second sampling showed no elevation of IFN-stimulated genes.

Neutrophil-stimulated genes were not overexpressed (figure 3B). The expression of a panel of other cytokine transcripts was not significantly altered (see online supplementary figure $\mathrm{S} 1$ ).

\section{DISCUSSION}

We report the case of two sisters with ADA2 deficiency and unusual clinical features at disease onset with initial normal cerebral MRI. Both presented with a previously undescribed mutation in CECR1, low ADA2 activity and the association of a high IFN signature on gene expression profiling, and a normal neutrophilic signature.

The clinical description of these two girls highlights the variable phenotype associated with ADA2 deficiency due to CECR1 mutations. ADA2 deficiency was first described in two majors articles, with fever, visceral and cutaneous lesions compatible with polyarteritis nodosa, and peripheral and central nervous system involvement. ${ }^{1}{ }^{2}$ While patient 1 in this report eventually demonstrated a comparable spectrum of disease features, she initially presented with an inflammatory myositis, which had not yet been described. ${ }^{2}$ Moreover, 

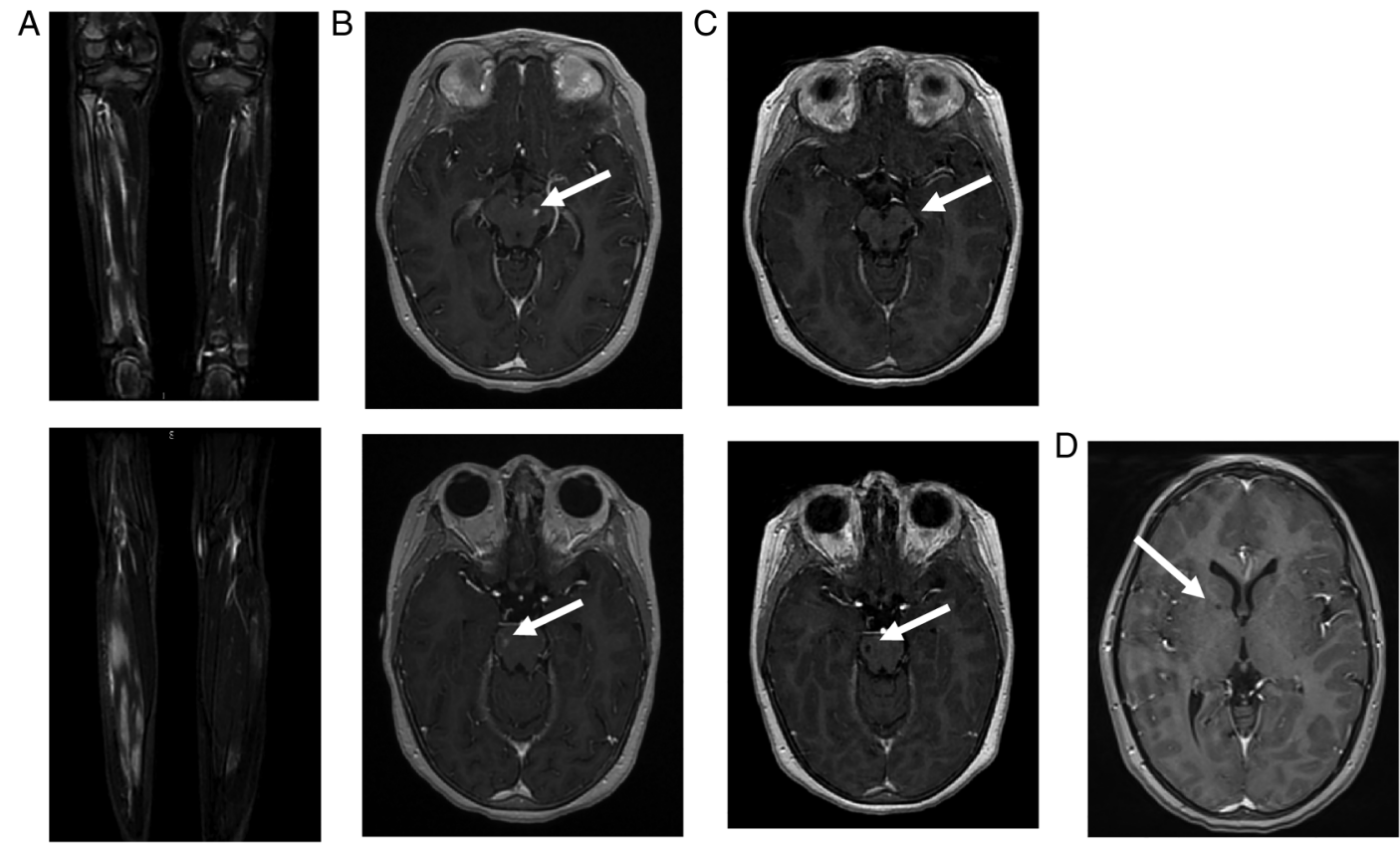

Figure 1 Imaging of patient 1. (A) Muscle MRI of both legs performed at the age of 5 years: coronal gadolinium-enhanced T1-weighted sequence at different levels, showing patchy inflammatory muscular lesions. (B) Cerebral MRI performed at the age of 7 years. Left. axial gadolinium-enhanced T1-weighted sequence, showing gadolinium-enhanced mesencephalic (up) and peduncular (down) lesions. Right. axial diffusion-weighted sequence showing mesencephalic (up) and peduncular (down) hyperintensities. (C) Cerebral MRI performed 4 months later: axial gadolinium-enhanced T1-weighted sequence showing evolution towards lacunar lesions (up: mesencephalic lesions/down: peduncular lesions). (D) Cerebral MRI performed 3 years later: gadolinium-enhanced T1-weighted sequences showing lacunar lesions located in the internal capsule.

repeated cerebral MRIs, with diffusion sequences, were normal in the acute period, which is most often associated with encephalopathy, suggestive probably of transient ischaemic strokes affecting medium and small arteries evolving later to a stroke with lacunar lesions. The clinical presentation of this patient's sister was different. While patients have been described with only cutaneous involvement, ${ }^{6} 7$ this is the first report of a child with early stroke and no other features beyond a persistent mild inflammatory syndrome, including no cutaneous features. Hence, ADA2 deficiency should be considered as a differential diagnosis of atypical myositis or unexplained neurological events in the context of persistent inflammation, even in the absence of
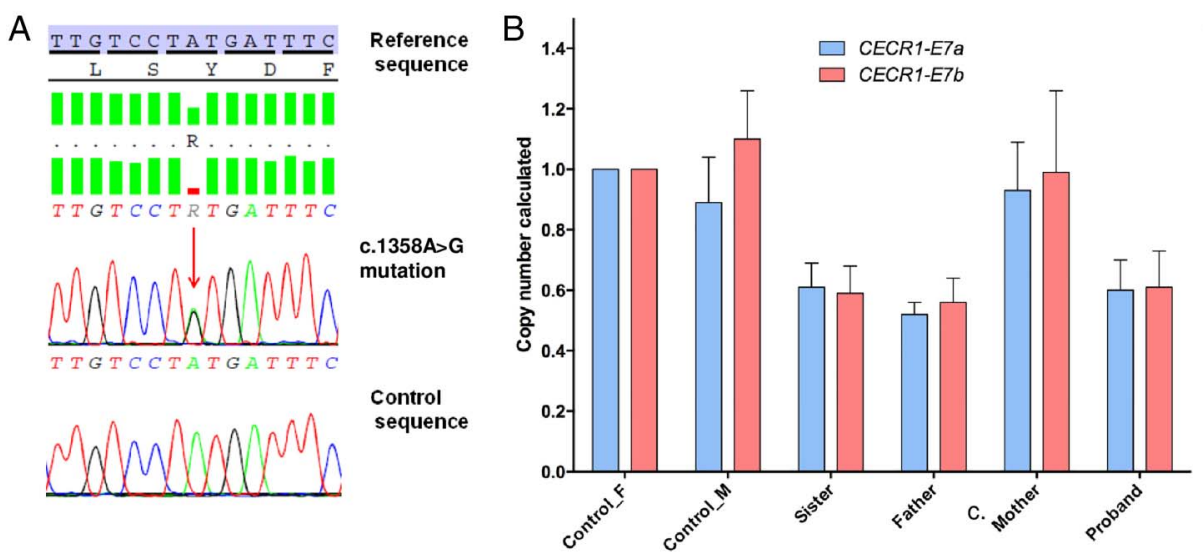

C

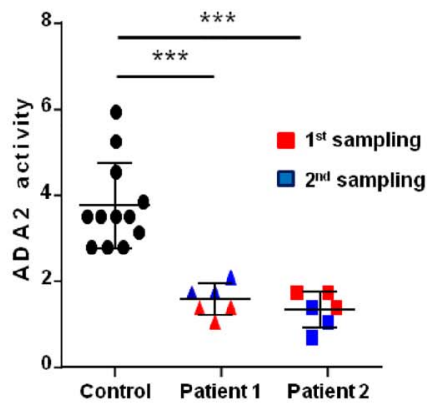

Figure 2 Illustration of the mutation and ADA2 activity. (A) Sanger sequencing analysis illustrating the point c.1358A $>G$ mutation which was identified in both sisters. (B) Reverse quantitative PCR (RQ-PCR, copy number calculated) illustrating the exon 7 deletion in the two sisters and in the father. CECR1-E7a and CECR1-E7b are two distinct amplicons encompassing exon 7 of CECR1 gene, to avoid a possible rare polymorphism in a primer hybridisation site. (C) CECR1 mutations result in a decrease in ADA2 activity in patient plasma. The figure shows the ADA2 activity in the plasma of the two patients tested on two occasions, compared with ADA2 activity in the plasma of four controls $\left({ }^{* \star *} \mathrm{p}<0.001\right)$. 
Figure 3 IFN and neutrophil gene expression studies. The $R Q$ value for each transcript is equal to the normalised fold change relative to a control. (A) Quantitative reverse transcription PCR of a panel of six IFN-1-stimulated genes in CECR1 mutation-positive sisters on two occasions. IFN score was 4.82 before treatment and 1.86 after treatment in patient 1 and 11.79 and 9.47 before treatment in patient 2. IFN-stimulated genes are variably overexpressed in the two patients. (B) The expression of a panel of neutrophil-stimulated genes was measured in patients 1 and 2 on two occasions. Neutrophil-stimulated gene expression was essentially similar to that in controls. IFN-1, interferon 1; NA, not applicable; $R Q$ value, relative quantification value.
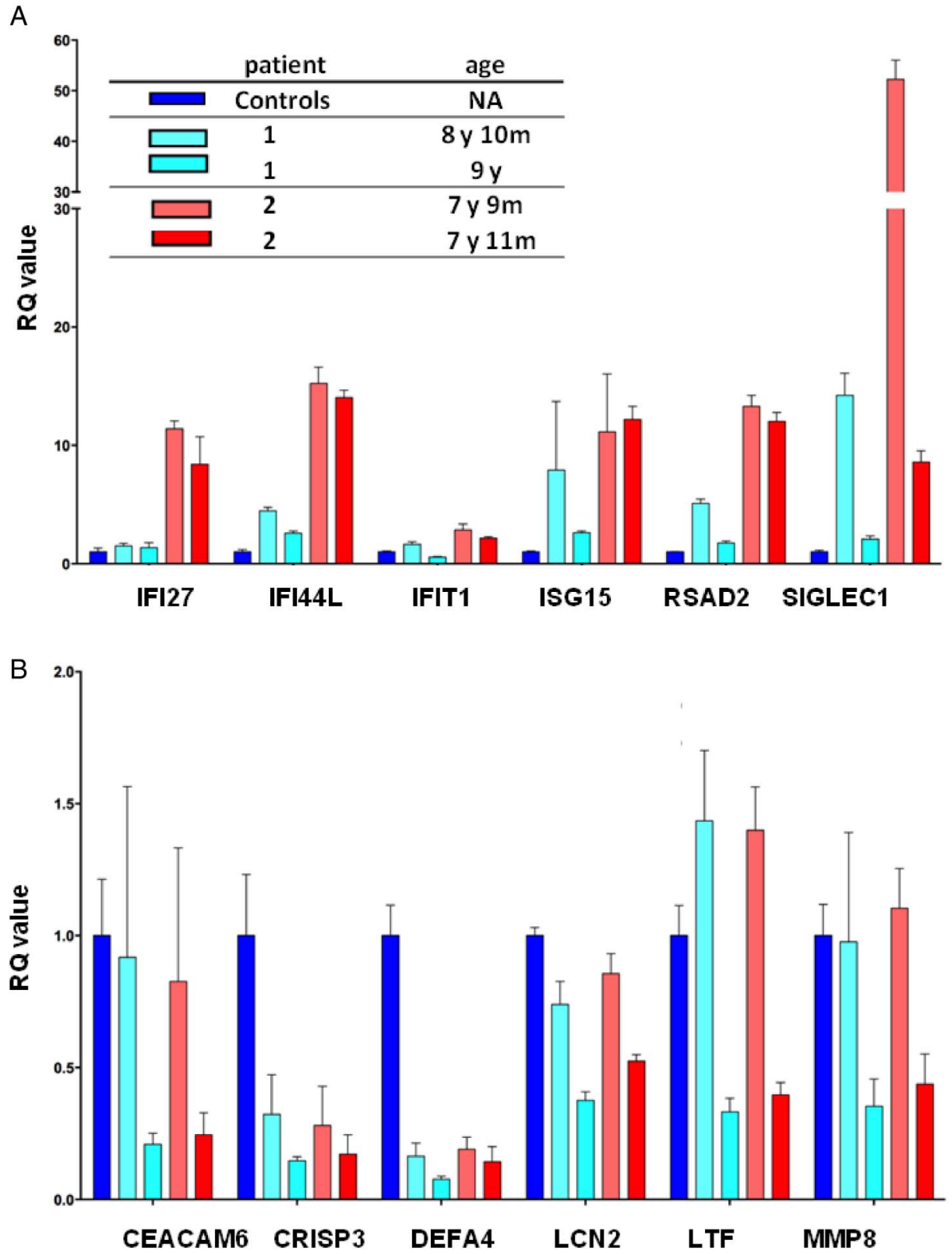

cutaneous involvement. ${ }^{6} 8$ Finally, our patients exhibited neither hypogammaglobulinaemia nor recurrent infections, even though there were low IgM and IgA levels and mild B cell lymphopenia, in accordance with the hypothesis that ADA2 deficiency may lead to a defect in memory B cells. ${ }^{9}$ Interestingly, both patients also had mild biological markers of autoimmunity (ANA/lupus anticoagulant), as already reported. ${ }^{12}$

While the p.Tyr453Cys mutation has been reported previously, we provide the first description of an exonic deletion of CECR1. This possibility should therefore be borne in mind when testing for CECR1 mutations. Some phenotype-genotype correlations have been tentatively suggested, in particular for the G47R mutation (which might be associated less frequently with cerebrovascular involvement). ${ }^{10}$ However, this report and others underline the fact that marked phenotypic variability can occur even in the same family. ${ }^{11}$
ADA2 enzymatic activity was reduced by almost $50 \%$ in both patients, although the clinical presentation was different. This observation indicates that ADA2 activity does not reflect the severity of the disease. ${ }^{12}$ We suggest that ADA2 activity dosage could be a complementary tool to diagnose ADA2 deficiency in cases where the clinical picture is suggestive and genetic testing is not conclusive (eg, in the presence of a missense variant of uncertain significance).

Gene expression analysis has already been performed in two patients with ADA2 deficiency. IFN-stimulated genes and neutrophil expressed gene overexpression have been suggested by Belot $e t a \tilde{l}$ to be relevant to the disease phenotype. Here, an upregulation of IFN-stimulated gene expression was demonstrated in both sisters, with the exception of the second sampling in the older sister. This might be partially explained by the fact that the patient did not have active disease at 
the time of sampling and was under anti-TNF treatment. However, it is unclear to what extent the disease activity and the treatment affect cytokine transcription profiles in this disease, a point that should certainly be addressed further in future studies.

Of further note, neutrophil-derived gene expression was not elevated. It was previously hypothesised that a neutrophilic signature might explain part of the ADA2 deficiency phenotype, with a suggested role for ADA2 as plasmatic regulator of peripheral blood mononuclear cell activation. ${ }^{3} 512$ The patients described here make that possibility less likely. Meanwhile, inflammatory cytokine expression assessment did not reveal any consistent disease correlations, in accordance with previous data. ${ }^{1}$

Regarding ADA2 deficiency treatment, more than 20 patients have previously received systemic therapy including anti-interleukin 1 (IL-1), without efficacy in most cases. Moreover, treatment with fresh frozen plasma and bone marrow transplantation have been proposed in regard to the protein deficiency. ${ }^{13}{ }^{14}$ Furthermore, it was also reported that treatment with anti-TNF and IL-6 blockade could lead to clinical improvement (and, in the case of the former, almost normalise a previously high neutrophil signature). ${ }^{5}{ }^{14}$ In our patients, shortterm response to anti-TNF therapy was excellent.

To conclude, screening for CECR1 mutations should be considered in patients with an atypical presentation of inflammatory disease, including myositis. Efforts to collect new cases, in particular through the Eurofever registry, may allow us to improve our disease knowledge, straddling the borders of autoinflammation and immunological deficiency. ${ }^{15}$ Importantly, although the pathological basis of this severe disease remains unclear, highly promising therapeutic strategies have already emerged.

\section{Author affiliations}

${ }^{1}$ Pediatric Immunology-Hematology and Rheumatology Unit, Institut Imagine, Hôpital Necker-Enfants Malades, Assistance Publique Hôpitaux de Paris, Paris, France

${ }^{2}$ Transversal Unit of Allergology and Rheumatology, CHRU Tours, Tours, France

${ }^{3}$ Laboratory of Rare and Autoinflammatory Genetic Diseases, CHRU, Montpellier, France

${ }^{4}$ INSERM UMR 1163, Laboratory of Neurogenetics and Neuroinflammation, Institut Imagine, Hôpital Necker, Paris Descartes-Sorbonne Paris Cité University, Paris, France

${ }^{5}$ Manchester Centre for Genomic Medicine, Institute of Human Development Faculty of Medical and Human Sciences, Manchester Academic Health Sciences Centre, University of Manchester, Manchester, UK

${ }^{6}$ Inserm U930, CNRS 2448, Tours, France

${ }^{7}$ Service Neuropédiatrie et Handicaps, CHRU de Tours, Tours, France

${ }^{8}$ Pediatric Emergency Unit, CHRU Tours, Tours, France

${ }^{9}$ Pediatric Neurology Department, National Referral Center for NeuroInflammatory Diseases in Children, Hôpitaux Universitaires Paris Sud, Assistance Publique-Hôpitaux de Paris and UMR 1184, Center for Immunology of viral infections and autoimmune diseases, University Paris Sud, Le Kremlin-Bicêtre, France
Acknowledgements YJC acknowledges the European Research Council (GA 309449: Fellowship), and a state subsidy managed by the National Research Agency (France) under the 'Investments for the Future' program (reference ANR-10-IAHU-01).

Contributors FU wrote the manuscript and followed the two children with YM for the rheumatology and the help of two neurologists EL and KD for the analyses of cerebral MRI. GS and IT performed the genetics analyses. GIR and MPR performed the ADA2 activity dosage and the interferon and neutrophil signature under the supervision of YJC. YJC also mostly participated in the correction of the manuscript. $P Q$ contributed to all the work and especially to the correction of the manuscript.

Competing interests None declared.

Patient consent Parental/guardian consent obtained.

Provenance and peer review Not commissioned; externally peer reviewed.

Data sharing statement No additional data are available.

Open Access This is an Open Access article distributed in accordance with the Creative Commons Attribution Non Commercial (CC BY-NC 4.0) license, which permits others to distribute, remix, adapt, build upon this work noncommercially, and license their derivative works on different terms, provided the original work is properly cited and the use is non-commercial. See: http:// creativecommons.org/licenses/by-nc/4.0/

\section{REFERENCES}

1. Zhou Q, Yang D, Ombrello AK, et al. Early-onset stroke and vasculopathy associated with mutations in ADA2. $N$ Engl J Med 2014;370:911-20.

2. Navon Elkan P, Pierce SB, Segel R, et al. Mutant adenosine deaminase 2 in a polyarteritis nodosa vasculopathy. $N$ Engl $J$ Med 2014;370:921-31.

3. Zavialov AV, Engström A. Human ADA2 belongs to a new family of growth factors with adenosine deaminase activity. Biochem $J$ 2005;391(Pt 1):51-7.

4. Poursharifi P, Saghiri R, Ebrahimi-Rad M, et al. Adenosine deaminase in patients with primary immunodeficiency syndromes: the analysis of serum ADA1 and ADA2 activities. Clin Biochem 2009;42:1438-43.

5. Belot $A$, Wassmer $E$, Twilt $M$, et al. Mutations in CECR1 associated with a neutrophil signature in peripheral blood. Pediatr Rheumatol Online J 2014;12:44.

6. Westendorp WF, Nederkoorn PJ, Aksentijevich I, et al. Unexplained early-onset lacunar stroke and inflammatory skin lesions: consider ADA2 deficiency. Neurology 2015;84:2092-3.

7. Gonzalez Santiago TM, Zavialov A, Saarela J, et al. Dermatologic Features of ADA2 deficiency in cutaneous polyarteritis nodosa. JAMA Dermatol 2015;151:1230-4.

8. Segel R, King MC, Levy-Lahad E. Mutant ADA2 in vasculopathies. $N$ Engl J Med 2014;371:481-2.

9. Schena F, Volpi S, Pastorino $\mathrm{C}$, et al. B cell characterization in ADA2 deficiency patients. Ann Rheum Dis 2015;74(Suppl 2):1132.

10. Batu ED, Karadag O, Taskiran EZ, et al. A case series of adenosine deaminase 2 deficient patients emphasizing genotype-phenotype correlations. Ann Rheum Dis 2015;74(Suppl 2):536.

11. Caorsi R, Grossi A, Insalaco A, et al. Prevalence of CECR 1 mutations in pediatric patients with polyarteritis nodosa, livedo reticularis and/or stroke. Ann Rheum Dis 2015;74(Suppl 2):835

12. Gakis C, Cappio-Borlino A, Pulina G. Enzymes (isoenzyme system) as homeostatic mechanisms the isoenzyme (ADA2) of adenosine deaminase of human monocytes-macrophages as a regulator of the 2'deoxyadenosine. Biochem Mol Biol Int 1998;46:487-94.

13. van Montfrans J, Zavialov A, Zhou Q. Mutant ADA2 in vasculopathies. N Engl J Med 2014;371:478.

14. Van Eyck L, Liston A, Wouters C. Mutant ADA2 in vasculopathies. N Engl J Med 2014;371:480.

15. Toplak N, Frenkel J, Ozen S, et al. An international registry on autoinflammatory diseases: the Eurofever experience. Ann Rheum Dis 2012;71:1177-82. 